The following pages constitute the final, accepted and revised manuscript of the article:

Petri Gudmundsson, BS, Erik Rydberg, Reidar Winter, Ronnie Willenheimer

"Visually estimated left ventricular ejection fraction by echocardiography is closely correlated with formal quantitative methods."

Int J Cardiol. 2005 May 25;101(2):209-12

Publisher: Elsevier.

Use of alternative location to go to the published version of the article requires journal subscription.

Alternative location:

http://dx.doi.org/ 10.1016/j.ijcard.2004.03.027 


\section{VISUALLY ESTIMATED LEFT VENTRICULAR EJECTION FRACTION BY ECHOCARDIOGRAPHY IS CLOSELY CORRELATED WITH FORMAL QUANTITATIVE METHODS}

Petri Gudmundsson, BS, Erik Rydberg, MD, PhD, Reidar Winter, MD, Ronnie Willenheimer, $\mathrm{MD}, \mathrm{PhD}$, senior lecturer

Address: Department of Cardiology Malmö University Hospital, University of Lund 20502 Malmö Sweden

Correspondence to: BMA Petri Gudmundsson

Malmö University Hospital, University of Lund 20502 Malmö

Sweden

Fax +46 40336209 , phone +4640331000

E-mail: petri.gudmundsson@skane.se 
Background Simpson ejection fraction(Simpson-EF), wall motion score index (WMSI), atrioventricular (AV) plane displacement (AVPD) and fractional shortening (FS) are all established formal echocardiographic methods for the assessment of left ventricular (LV) systolic function. Visually estimated (eyeballing) EF ejection fraction may be considered somewhat more subjective, although shown to correlate well with radionuclide ventriculography. We aimed to explore if echocardiographic eyeballing-EFeyeballing ejection fraction is comparable to formal methods for the evaluation of LV left ventricular systolic function.

Methods We assessed 89 consecutive patients after myocardial infarction $(n=54)$ or before coronary angiography $(n=35)$. Eyeballing-EFEyeballing ejection fraction and WMSI wall motion score index were evaluated in the long-axis, short-axis and apical 4- and 2-chamber views. Simpson-EF ejection fraction and AV plane displacement were assessed in the apical views. Fractional Sshortening was measured in the parasternal long-axis view. The respective systolic function measurements were in each patient made at different time points by a single investigator, masked to prior results.

Results All formal methods correlated significantly with eyeballing-EF eyeballing ejection fraction ( $\mathrm{p}<0.001)$ : AVPDAV plane displacement, $\mathrm{R}=0.647$; FS, $\mathrm{R}=0.684$; 4-chamber Simpson EFejection fraction, $\mathrm{R}=0.857$; biplane Simpson-EF ejection fraction, $\mathrm{R}=0.898$; and WMSIwall motion score index, $\mathrm{R}=0.919$. 
Conclusion Eyeballing-EF Eyeballing ejection fraction correlated closely with all formal methods and the correlation coefficient improved with the reliability of the formal method. This finding is in concordance with prior studies, indicating thateyeballing-EF eyeballing ejection fraction may be the most accurate echocardiographic method for the assessment of LV left ventricular systolic function. Since it is readily and quickly performed, eyeballing-EF eyeballing ejection fraction could be used for routine echocardiography instead of formal methods.

Keywords: Echocardiography, Systolic function, Visual ejection fraction, Eyeballing 


\section{INTRODUCTION}

Left ventricular systolic function can be assessed by echocardiography and expressed as ejection fraction, wall motion score index, fractional shortening or atrioventricular (AV) plane displacement. These are all important prognostic measurements in patients with chronic heart failure or myocardial infarction and have shown good correlation with the gold standard method, radionuclide ventriculography [1,2,3,4].

Compared to visual quantification, formal quantitative measurements of left ventricular ejection fraction, such as single- or biplane Simpson ejection fraction, may be considered superior echocardiographic methods for clinical and scientific use, because these methods may appear to be little influenced by subjectivity. But these methods are influenced by subjectivity, since the decision about were to mark the endocardial borders is subjective. Furthermore, besides being time consuming, the endocardial border tracing is sometimes difficult to perform, especially in patients with poor image quality, since it is performed on frozen images [4]. Consequently, the limits of agreement versus radionuclide examination are quite wide for these methods $[4,5,6,7]$. Wall motion score index might be easier to perform in patients with poor image quality, since the segments are evaluated on moving images, but the method is still more time consuming than visual estimation of global ejection fraction. The wall motion score index method is also subjective.

Compared to formal echocardiographic methods for the evaluation of left ventricular ejection fraction, visual estimation (eyeballing) can be done faster and is often easier to perform, even in patients with poor image quality. This method has also shown good correlation with the gold standard, radionuclide examination, and formal echocardiographic methods in prior studies $[7,8,9,10,11]$. However, the value of eyeballing ejection fraction is still questioned by some and may differ between echocardiographic laboratories. Furthermore, it has not been properly evaluated using second harmonic imaging. Therefore, we aimed to compare 
eyeballing ejection fraction with established, formal echocardiographic methods for the evaluation of left ventricular ejection fraction, using modern equipment with second harmonic imaging.

\section{MATERIALS and METHODS}

The study conforms to the ethical guidelines of the 1975 Declaration of Helsinki.

\section{PATIENTS}

We included 89 consecutive adult patients referred to the echocardiography laboratory at the Department of Cardiology, Malmö University Hospital, with a primary catchment area of 250 000 inhabitants. Mean age was $65 \pm 11$ years (range 44-85) and 61 (69 \%) of the 89 patients were men. The patients were included either within a week after an acute myocardial infarction ( $\mathrm{n}=54)$ or within two weeks before a planned coronary angiography in patients with stable angina $(\mathrm{n}=35)$. Since atrial fibrillation has been shown to decrease AV plane displacement disproportionately to left ventricular ejection fraction [12, 13], only patients in sinus rhythm were included in the present study.

\section{ECHOCARDIOGRAPHIC EXAMINATION}

The echocardiography examinations were performed by either of two investigators using an Agilent Sonos 5500 system and the S3 or S4 transducer. Parasternal and apical views were obtained with the patient in a left lateral recumbent position. Images were acquired during silent respiration or end-expiratory apnea. Using the Enconcert digital workstation, the respective systolic function measurements were in each patient performed at different time points, off line, by the same single investigator, masked to prior measurements. Eyeballing ejection fraction and wall motion score index were evaluated from 2D images in the longaxis, short-axis and apical 4- and 2-chamber views. A value of left ventricular ejection 
fraction was then determined from these views. Wall motion score index was determined according to the 16-segment model. Each segment was given a score: -1 , dyskinetic; 0, akinetic; 1, hypokinetic; 2, normokinetic; 3, hyperkinetic. All segment scores were then added and the sum was divided by the number of segments that could be scored, to get the wall motion score index. Single 4-chamber left ventricular ejection fraction and biplane Simpson left ventricular ejection fraction were measured in the apical views by tracing of the endocardial borders at end diastole and end systole. The digital workstation then calculated the left ventricular ejection fraction. AV plane displacement was assessed by twodimensionally guided M-mode echocardiography in the 4- and 2-chamber views, as described previously $[4,14,15]$. Fractional shortening was calculated from 2D measurements of the left ventricular end diastolic and end systolic diameters in the parasternal long-axis view.

\section{STATISTICS}

Simple linear regression analysis was used for assessment of correlations between continuous variables. Data are expressed as mean \pm SD. P-values $<0.05$ were considered significant.

\section{RESULTS}

Mean values of the respective methods for the assessment of left ventricular systolic function were: eyeballing ejection fraction, $51.85 \pm 14.90 \%$; AV plane displacement, $11.07 \pm 2.70$ mm; fractional shortening, $0.32 \pm 0.10$; single 4-chamber Simpson ejection fraction, $52.54 \pm$ $13.45 \%$; biplane Simpson ejection fraction, $54.31 \pm 14.33 \%$; and wall motion score index, $1.625 \pm 0.400$ units. In simple regression analysis all formal methods correlated significantly with eyeballing ejection fraction $(\mathrm{p}<0.001)$ : AV plane displacement, $\mathrm{R}=0.647$; fractional shortening, $\mathrm{R}=0.684$; single 4-chamber Simpson ejection fraction, $\mathrm{R}=0.857$ (Fig 1a); biplane Simpson ejection fraction, $\mathrm{R}=0.898$ (Fig 1b); and wall motion score index, $\mathrm{R}=0.919$ (Fig 1c). Table 1 depicts the intra-individual variability for the various methods. 


\section{DISCUSSION}

In the present study, eyeballing ejection fraction correlated significantly with all formal quantitative methods for the evaluation of left ventricular systolic function. Both wall motion score index and biplane Simpson ejection fraction have been shown to correlate closely with radionuclide ventriculography, more closely than single 4-chamber Simpson ejection fraction, fractional shortening and AV plane displacement $[7,16,17]$. Thus, in the present study, the correlation between eyeballing ejection fraction and the respective formal quantitative method improved with the reliability of and the number of views used by the formal method. This finding is in concordance with prior studies indicating that eyeballing ejection fraction may be the most accurate echocardiographic method for the assessment of left ventricular ejection fraction [7,9-11]. The fact that modern equipment with second harmonic imaging was used did not seem to affect the relation between eyeballing ejection fraction and the other methods $[7-11]$

It may be suggested that the accuracy of eyeballing ejection fraction is dependent on the skill of the examiner. This is part of the reason why we wanted to examine this relationship in our laboratory. However, our results are in concordance with those shown by other groups, indicating that eyeballing ejection fraction commonly can be used with a high level of accuracy [7,9-11]. Furthermore it has been shown in a study by Akinboboye et al [18] that eyeballing ejection fraction is a method easy to learn. If a person with no previous experience of echocardiography, after each evaluation is given instant feedback from a gold standard, about 60 evaluations are required to achieve the same accuracy as an experienced echocardiographer. Due to this, our results could probably be reproduced by anyone who receives formal practice and works regularly with echocardiography. Furthermore, a systematic review has recently shown that the limits of agreement were similar for Simpson 
ejection fraction, wall motion score index and eyeballing ejection fraction [19]. That study also showed that none of these methods appeared to systematically under- or overestimate left ventricular ejection fraction. The measurement variability in the present study was lower compared to what was reported in the systematic review [19], which probably is due to the use of second harmonic and the fact that all measurement were made by a single investigator in our study.

The results of the present and prior studies suggest that eyeballing ejection fraction could be accepted for scientific use, provided that the variability of eyeballing ejection fraction in the echocardiography laboratory is low. Indeed, eyeballing ejection fraction seems to be more accurate for the evaluation of left ventricular ejection fraction than fractional shortening, AV plane displacement and single plane Simpson ejection fraction, in patients after myocardial infarction or with angina pectoris, since eyeballing ejection fraction correlated better with the echocardiographic methods that correlate best with the gold standard.

Eyeballing ejection fraction and wall motion score index are both subjective methods that evaluate left ventricular contractility on moving images. In the present study, both methods were evaluated by the same person, which could have introduced some bias. However, the respective evaluations were done at different time points in each patient and the investigator was always masked to the results of prior measurements. Furthermore, biplane Simpson ejection fraction, which was measured on frozen images, correlated almost as closely with eyeballing ejection fraction. Thus, this type of bias is unlikely to explain the good correlations between eyeballing ejection fraction and formal measurements of left ventricular ejection fraction observed in the present study. Bias based on the visual impression of the left ventricular ejection fraction is however probably impossible to completely avoid. Even left ventricular ejection fraction based on tracing of the endocardial borders is affected by the 
visual impression, which is likely the case in everyday clinical practice as well as in scientific studies. However, this probably only increases the precision of these formal measurements, since the eyeballing ventricular ejection fraction repeatedly has been show to correlate more closely with radionuclide ventriculography than any formal echocardiographic measurement of left ventricular ejection fraction [7,9-11].

There might be situations when it is of great importance to notice small changes in left ventricular function, e.g. in response to short term pharmacological intervention. In such cases the used method has to be very reliable. In these situations we recommend the use of AV plane displacement, which shows very low variability.

\section{CONCLUSION}

Eyeballing ejection fraction correlated significantly with all formal methods for the evaluation of left ventricular systolic function. The correlation with eyeballing ejection fraction improved with the reliability of and the number of views used by the formal quantitative method. This finding is in concordance with prior studies, indicating that eyeballing ejection fraction may be the most accurate echocardiographic method for the assessment of left ventricular systolic function. Since it is readily and quickly performed, eyeballing ejection fraction could be used for routine purposes in clinical practise, instead of the formal quantitative methods, which are more time consuming. If the echocardiography laboratory has a proven low variability of the measurement of eyeballing ejection fraction, this method may be used for scientific purposes, since it is as least as accurate as formal, “objective” echocardiographic methods. 


\section{REFERENCES}

[1] Basilico FC, Folland ED, Karaffa S, Tow DE, Parisi AF. Non-invasive measurement of left ventricular function in coronary artery disease. Comparison of first pass radionuclide ventriculography, M-mode echocardiography, and systolic time intervals. Br Heart J 1981; 45: 369-75.

[2] Galasko GI, Basu S, Lahiri A, Senior R. A prospective comparison of echocardiographic wall motion score index and radionuclide ejection fraction in predicting outcome following acute myocardial infarction. Heart 2001; 86: 271-6.

[3] Kober L, Torp-Pedersen C, Carlsen J, Videbaek R, Egeblad H. An echocardiographic method for selecting high risk patients shortly after acute myocardial infarction, for inclusion in multi-centre studies (as used in the TRACE study). TRAndolapril Cardiac Evaluation. Eur Heart J 1994; 15: 1616-20.

[4] Willenheimer R, Cline C, Erhardt L, Israelsson B. Left ventricular atrioventricular plane displacement: An echocardiographic technique for rapid assessment of prognosis in heart failure. Heart 1997; 78: 230-6.

[5] Folland ED, Parisi AF, Moynihan PF, Jones DR, Feldman CL, Tow DE. Assessment of left ventricular ejection fraction and volumes by real-time, two-dimensional echocardiography. A comparison of cineangiographic and radionuclide techniques.Circulation 1979; 60: 760-6.

[6] Starling MR, Crawford MH, Sorensen SG, Levi B, Richards KL, O’Rourke RA. Comparative accuracy of apical biplane cross-sectional echocardiography and gated equilibrium radionuclide angiography for estimating left ventricular size and performance Circulation. 1981; 63: 1075-84.

[7] Jensen-Urstad K, Bouvier F, Höjer J et al. Comparison of different echocardiographic methods with radionuclide imaging for measuring left ventricular ejection fraction during 
acute myocardial infarctiontreated by thrombolytic therapy. Am J Cardiol 1998 ;81: 53844.

[8] van Royen N, Jaffe CC, Krumholz HM et al. Comparison and reproducibility of visual echocardiographic and quantitative radionuclide left ventricular ejection fractions. Am J Cardiol 1996; 77: 843-50.

[9] van 't Hof AW, Schipper CW, Gerritsen JG, Reiffers S, Hoorntje JC. Comparison of radionuclide angiography with three echocardiographic parameters of left ventricular function in patients after myocardial infarction. Int J Card Imaging 1998; 14: 413-8.

[10] Mueller X, Stauffer JC, Jaussi A, Goy JJ, Kappenberger L. Subjective visual echocardiographic estimate of left ventricular ejection fraction as an alternative to conventional echocardiographic methods: comparison with contrast angiography. Clin Cardiol 1991; 14: 898-902.

[11] Amico AF, Lichtenberg GS, Reisner SA, Stone CK, Schwartz RG, Meltzer RS. Superiority of visual versus computerized echocardiographic estimation of radionuclide left ventricular ejection fraction. Am Heart J 1989; 1181: 259-65.

[12] Alam M, Thorstrand C. Left ventricular function in patients with atrial fibrillation before and after cardioversion. Am J Cardiol 1992; 69: 694-6.

[13] Emilsson K, Wandt B. The relation between ejection fraction and mitral annulus motion before and after direct-current electrical cardioversion.

Clin Physiol. 2000; 20: 218-24.

[14] Willenheimer R, Erhardt L, Cline C, Rydberg E, Israelsson B. Prognostic significance of changes in left ventricular systolic function in elderly patients with congestive heart failure. Coronary Artery Disease 1997; 8: 711-7.

[15] Willenheimer R, Israelsson B, Cline C, Rydberg E, Broms K, Erhardt L. Left atrioventricular plane displacement (AVPD) is related to both systolic and diastolic left ventricular performance in patients with chronic heart failure. Eur Heart J 1999; 20: 612 
[16] Bellenger NG, Burgess MI, Ray SG et al. Comparison of left ventricular ejection fraction and volumes in heart failure by echocardiography, radionuclide ventriculography and cardiovascular magnetic resonance; are they interchangable? Eur Heart J 2000; 21: 138796.

[17] Vandenbossche JL, Kramer BL, Massie BM, Morris DL, Karliner JS. Two-dimensional echocardiographic evaluation of the size, function and shape of the left ventricle in chronic aortic regurgitation: comparison with radionuclide angiography. J Am Coll Cardiol 1984; 4: 1195-206.

[18] Akinboboye O, Sumner J, Gopal A et al. Visual estimation of ejection fraction by twodimensional echocardiography: the learning curve. Clin Cardiol 1995; 18: 726-9.

[19] McGowan JH, Cleland JG. Reliability of reporting left ventricular systolic function by echocardiography: a systematic review of 3 methods. Am Heart J 2003; 146: 380-2. 


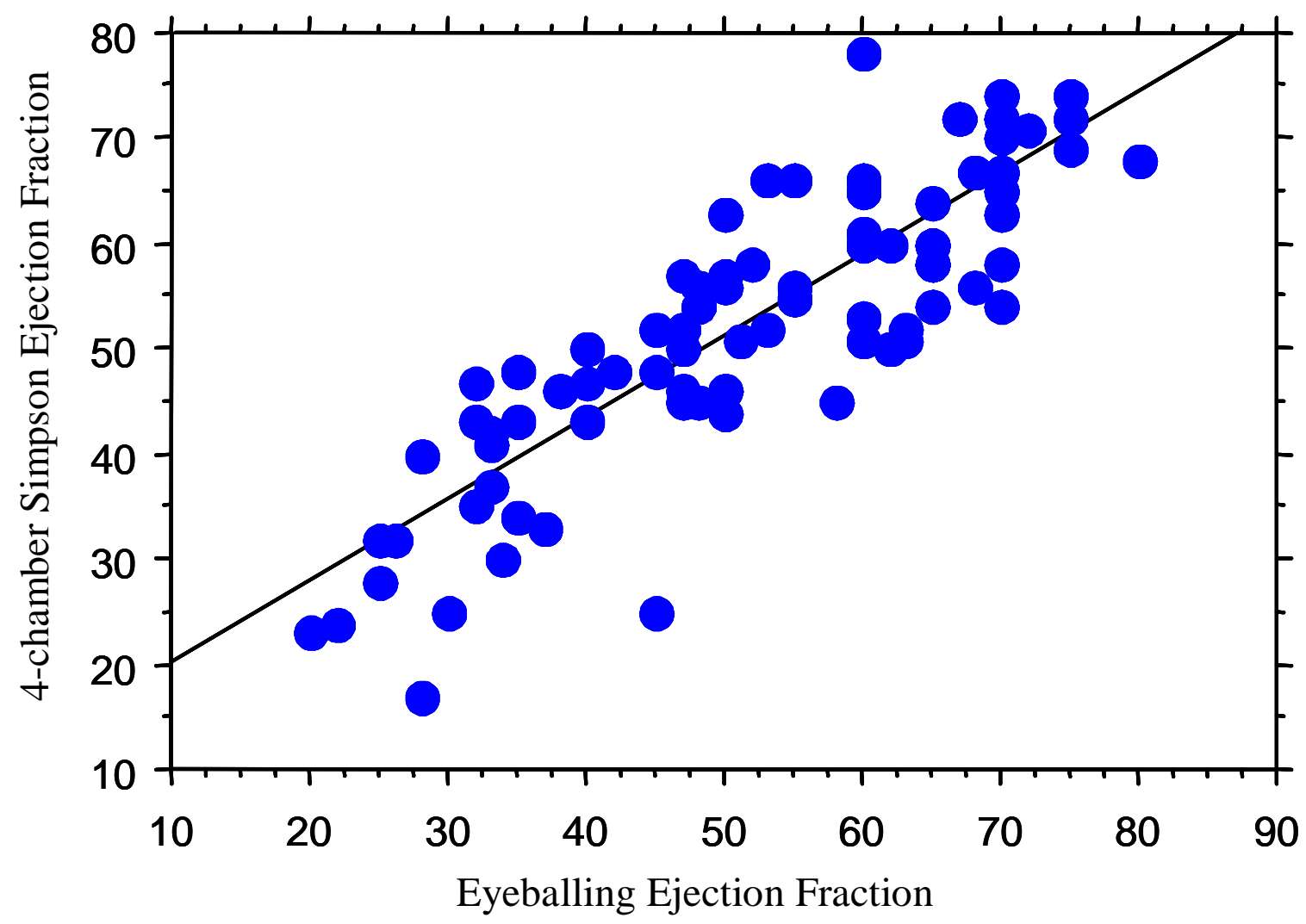

Fig. 1a. Correlation plot: Eyeballing Ejection Fraction versus 4-chamber Simpson Ejection Fraction $(\mathrm{R}=0.857)$. 


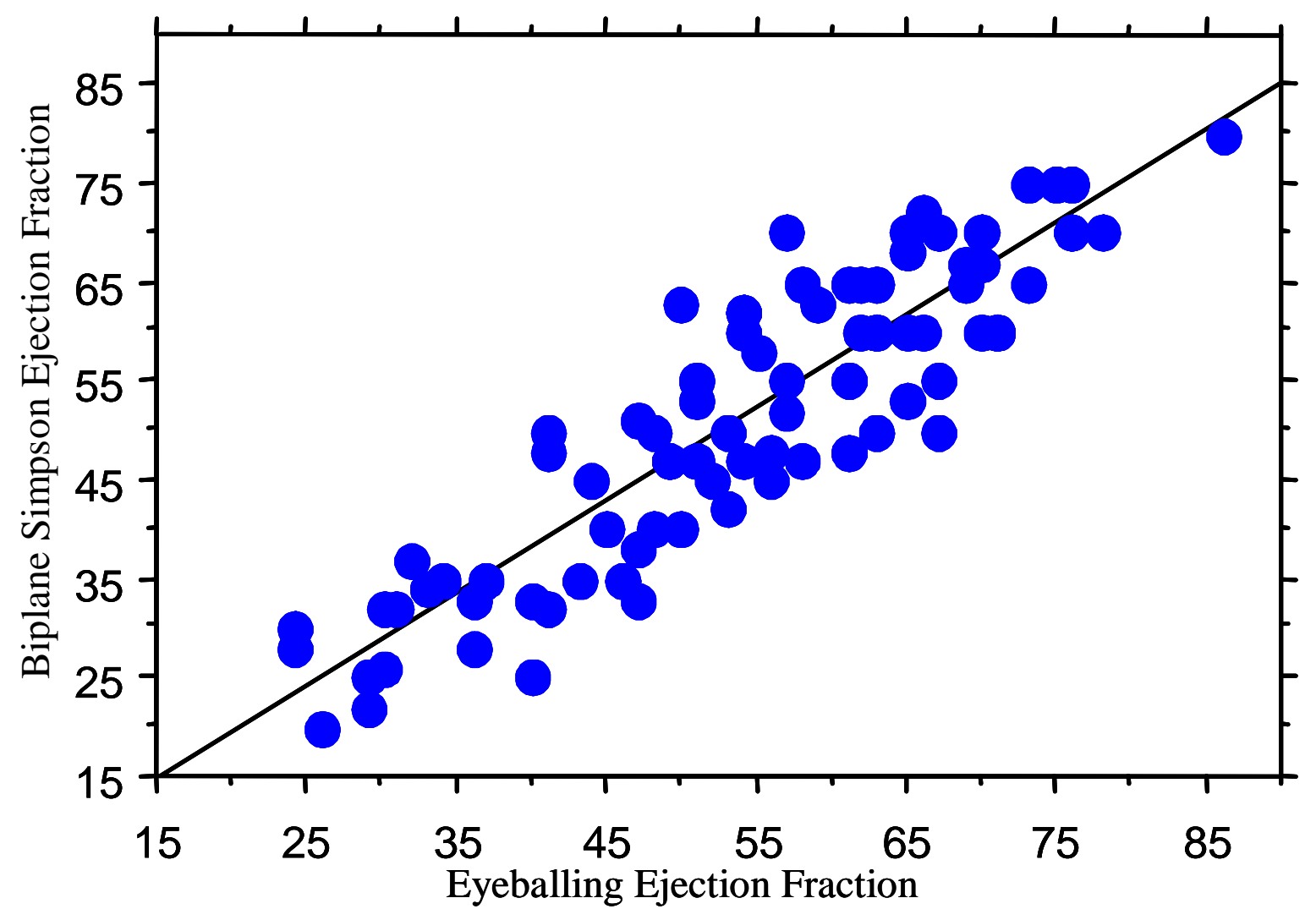

Fig. 1b. Correlation plot: Eyeballing Ejection Fraction versus Biplane Simpson Ejection Fraction $(\mathrm{R}=0.898)$. 


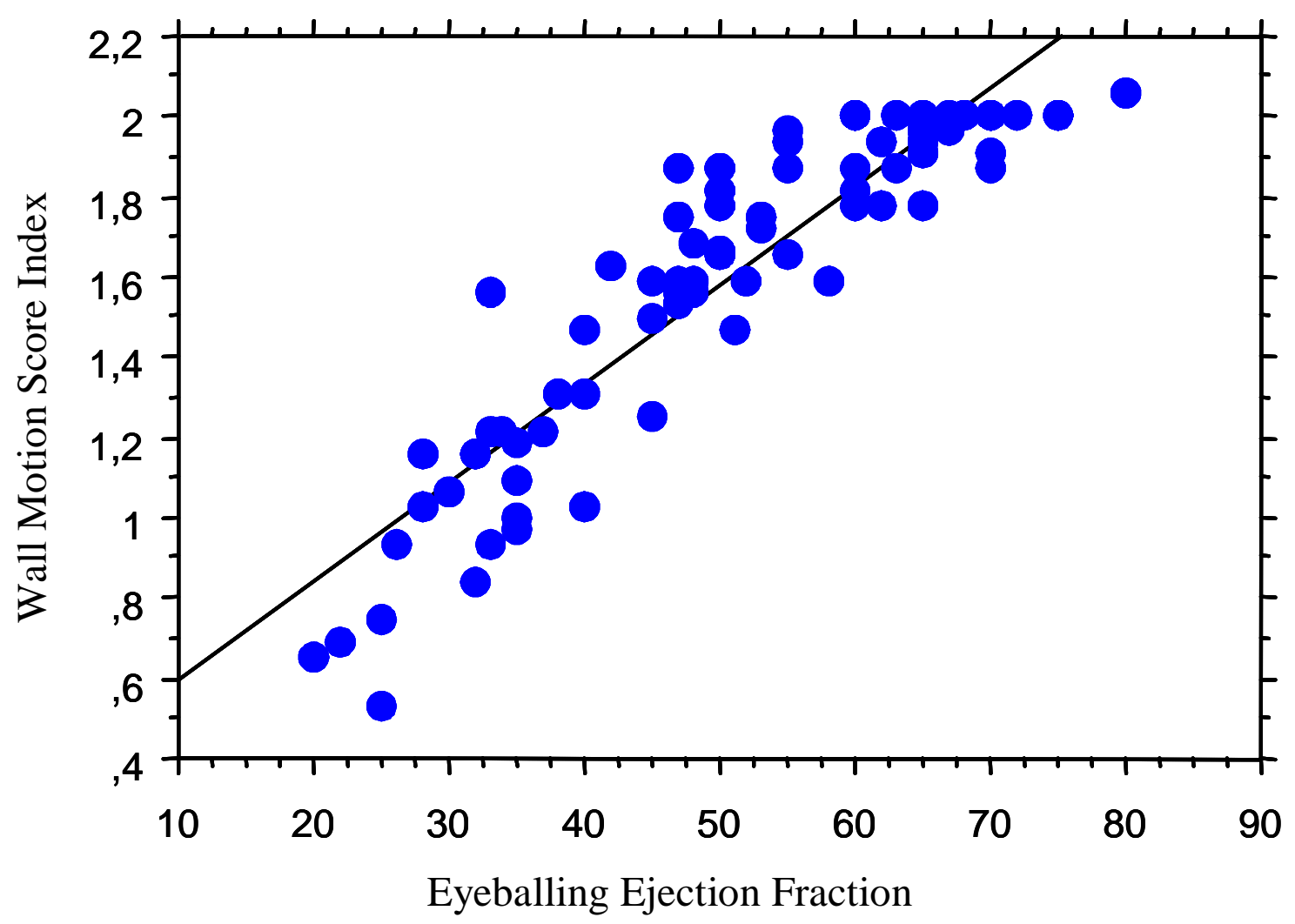

Fig. 1c. Correlation plot: Eyeballing Ejection Fraction versus Wall Motion Score Index $(\mathrm{R}=0.919)$. 
Table 1. Intra-individual variability for the different echocardiographic methods.

\begin{tabular}{|lcc|}
\hline & Mean variability (\%) & Range (\%) \\
\hline Eyeballing Ejection Fraction & 7.0 & $0-16.4$ \\
\hline Wall Motion Score Index & 8.7 & $0-35$ \\
\hline Biplane Simpson Ejection Fraction & 11.6 & $0-45.9$ \\
\hline Single 4-chamber Simpson Eejction Fraction & 9.3 & $0-24.4$ \\
\hline Fractional Shortening & 7.9 & $0.6-26.4$ \\
\hline AV Plane Displacement & 2.0 & $0-6$ \\
\hline
\end{tabular}

